\title{
Large left atrial cavernous hemangioma, a case report
}

\author{
Jian Xu, Hulin Piao, Duo Wang, Yong Wang, Bo Li, Tiance Wang, Zhicheng Zhu, Dan Li, Rihao Xu* (D) and \\ Kexiang Liu*
}

\begin{abstract}
Background: Cardiac cavernous hemangiomas are extremely rare and usually difficult to be diagnosed for being asymptomatic.

Case presentation: An asymptomatic 56-year-old woman was hospitalized due to a heart mass found by chest computed tomography (CT) during her annual physical examination. Coronary computed tomography angiography (CTA) disclosed a tumorous lesion, located in the left atrial roof and extended to the posterior wall of the aortic root and surrounding the left main coronary artery. However, there was no communicating branches between the hemangioma and coronary artery and no coronary artery stenosis. The tumor was excised with low-frequency electrocautery under cardiopulmonary bypass. The histopathological examination indicated the mass a cavernous hemangioma. The patient was discharged with an uneventful recovery.

Conclusions: Here we presented a rare case of successfully excision of a cavernous hemangioma involving the left atrial roof and left coronary artery. We advocate adequate exposure and complete surgical excision with lowfrequency electrocautery to avoid remnants and excessive resection.
\end{abstract}

Keywords: Cavernous hemangioma, Cardiac benign tumor

\section{Background}

Cardiac hemangiomas are extremely rare benign vascular tumors of the heart, with an incidence of less than $0.03 \%$ at autopsy [1]. Histologically, cardiac hemangiomas can be classified into three categories: capillary, cavernous, and arteriovenous hemangiomas [2]. Of these, cardiac cavernous hemangiomas are exceptionally rare, and seldom involving the left atrial and coronary artery. Surgical excision should be performed as early as possible after diagnosis [3]. To our knowledge, only 3 references of surgically treated left atrial roof cavernous hemangiomas have been reported [4-6]. We reported a cavernous hemangioma located in the left atrial roof and involving the left coronary artery.

\section{Case presentation}

An asymptomatic 56-year-old female was hospitalized because of a cardiac mass noted by a chest computed tomography $(\mathrm{CT})$ scan during a physical examination.

\footnotetext{
* Correspondence: xu6mouse789@sina.com; kxliu64@hotmail.com Department of Cardiovascular Surgery, Second Hospital of Bethune, Jilin University, No. 218 Ziqiang Street, Changchun 130022, China
}

Preoperative transthoracic echocardiography (TTE) revealed an uneven hyperechoic mass, measuring $81 \mathrm{~m} \times 38$ $\mathrm{mm}$, located in the left atrium. The mass attached to the interatrial septum adjacent to the mitral annulus but was not hampering blood flow of mitral valve. Preoperative coronary computed tomography angiography (CTA) showed a relatively low-intensity mass $(78 \mathrm{~mm} \times 42 \mathrm{~mm})$ in the left atrial roof and extended to the posterior wall of the aortic root. Coronary CTA revealed no communicating branches between the hemangioma and coronary artery or coronary artery stenosis (Fig. 1). Thus, the surgery was performed via a median sternotomy under cardiopulmonary bypass $(\mathrm{CPB})$. After the pericardium was incised, the tumor was found to cover the left atrial roof and extend to the posterior wall of the aortic root (Fig. 2). We successfully excised the tumor with low-frequency electrocautery from the left atrium to the aortic root without injury the coronary artery and the left atrial wall. Left atrium was opened to inspect the invasion status. Its endocardium was smooth and clear of invasion. After heart resuscitation, the patient weaned easily off bypass. On gross inspection, the tumor was a $7 \mathrm{~cm} \times 4 \mathrm{~cm} \times 3 \mathrm{~cm}$, 


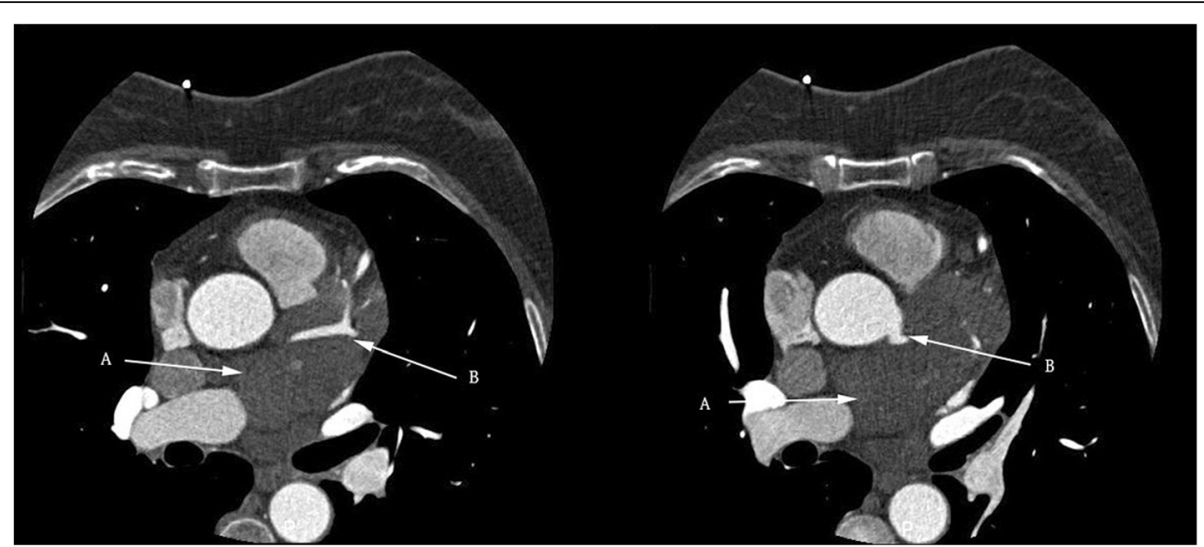

Fig. 1 Coronary CTA. A large low-density mass was located in the epicardium of the left atrial roof with ambiguous boundaries. The left coronary artery was not affected (A. Tumor, B. Left coronary artery)

elastic, soft and reddish-brown mass (Fig. 3). Histological examination after hematoxylin-eosin staining demonstrated a tumor comprised multiple dilated vascular channels with endothelial cell lining (Fig. 4). Cells lining in the vessels stained positive with CD31, CD34(Fig. 5), Indicating their endothelial origin. Cytologic examination revealed the presence of numerous mesenchymal and inflammatory cells, and absence of malignant cells.

The patient was discharged 5 days after the operation with an uneventful recovery. Telephone following-up is conducted every 3 months after discharge, the patient claimed no obvious discomfort. TTE 6 months after surgery showed no relapse of the cavernous hemangioma.

\section{Discussion}

Cavernous hemangioma is a rare disease. The clinical presentations of the cavernous hemangiomas of the heart are nonspecific and variable according to the location of the tumor and the resultant cardiac hemodynamic consequences. Manifestations of the symptomatic patients are arrhythmias, heart failure, embolic episodes, pericardial effusion, cardiac tamponade, myocardial ischemia and sudden death [3]. Most patients are asymptomatic, in our case, the patient was diagnosed through imaging during routine physical examination while no complaint was expressed.

Imaging methods are widely applied for diagnosing cardiovascular anomalies and cardiac tumor. Echocardiography is typically used to identify a cardiac mass; but $\mathrm{CT}$ and magnetic resonance imaging (MRI) are superior to echocardiography for further characterizing cardiac hemangiomas [7]. Coronary artery angiography can occasionally establish the diagnosis of hemangioma by its

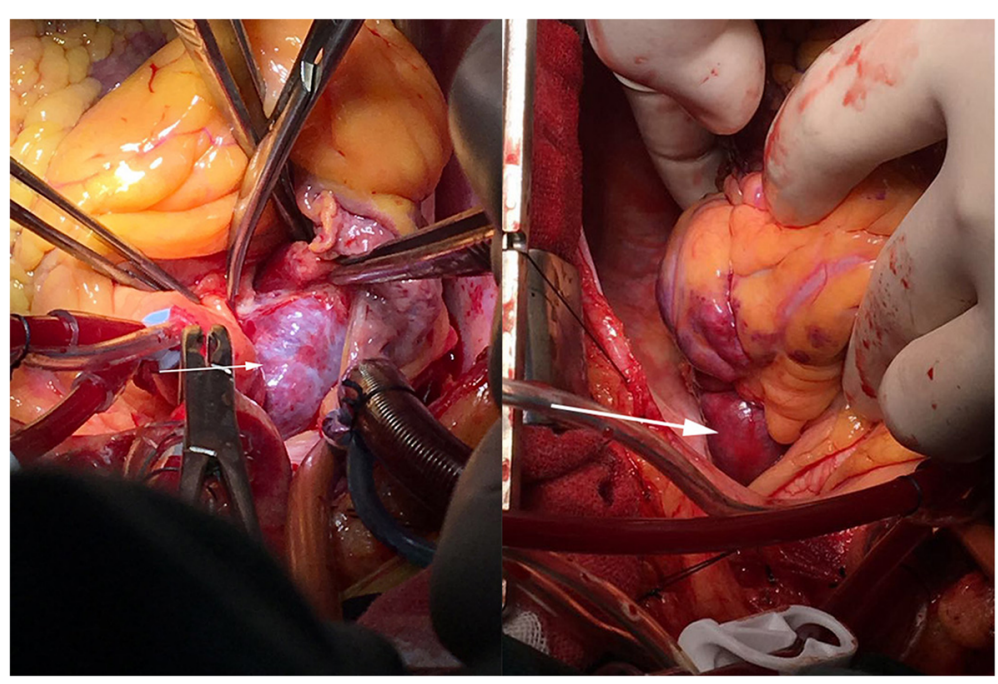

Fig. 2 Intraoperative view of the tumor 


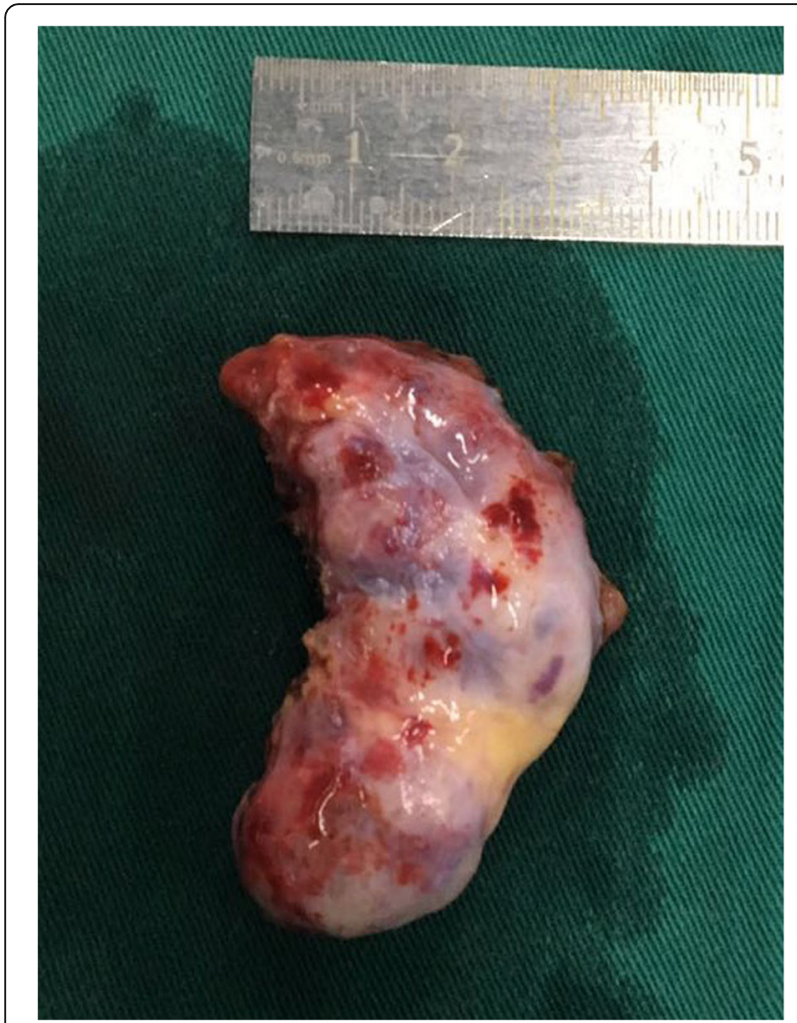

Fig. 3 The gross inspection of the tumor. It was a $7 \mathrm{~cm} \times 4 \mathrm{~cm} \times 3$ $\mathrm{cm}$, elastic, soft and reddish-brown mass

characteristic tumor blushing [5]. Definitive diagnosis was made based on postoperative pathological findings. In this case, Preoperative transthoracic echocardiography (TTE) revealed an intracardiac tumor. While the location of the lesion was determined poorly. Coronary CTA was administrated and a tumorous lesion was disclosed and located in left atrial roof. Coronary CTA is an useful method to assess the extent of cardiac hemangiomas

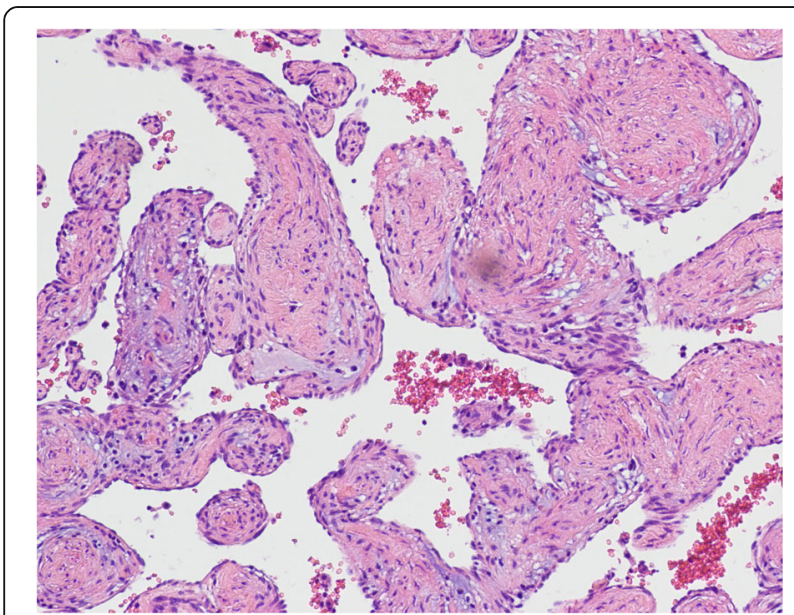

Fig. 4 The histopathological examination revealed cavernous hemangioma

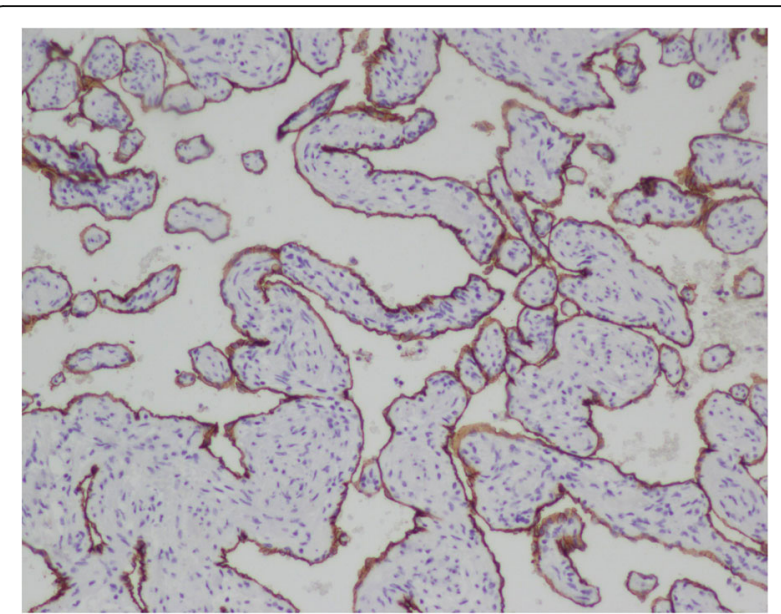

Fig. 5 Immunohistochemical staining of the cavernous hemangioma. Cells lining the vessels stained positive with CD31, CD34, supporting their endothelial origin

and the vascular supply of coronary arteries. It also has a high evaluability and sensitivity in detecting coronary stenosis.

Surgical excision should be performed as early as possible once diagnosed, especially in symptomatic cases [3]. Median sternotomy with cardiopulmonary bypass is universally required. Surgical resection aided by thoracoscopy or mini-thoracotomy can also be taken into consideration according to the mass location and adhesions situation. In this case, the cardiac cavernous hemangioma located in the left atrial roof, extended to the posterior wall of the aortic root and surrounding the left main coronary artery. Median sternotomy was conducted under CPB to provides adequate exposure. The mass extended widely and involved the left coronary artery, which has not been reported in other cases yet. Coronary artery bypass grafting (CABG) and reconstruction of the left atrial wall may need to be conducted in invasive cases $[6,8]$. The tumor was carefully excised with low-frequency electrocautery. However, despite of extensive compression and distortion of surrounding structures, the tumor was excised completely without injuring the left coronary artery and left atrial wall.

\section{Conclusions}

In this rare case, our center conducted the excision of a cavernous hemangioma involving the left atrial roof and left coronary artery. We advocate adequate exposure and complete surgical excision with low-frequency electrocautery to avoid remnants and excessive resection.

\section{Abbreviations}

CABG: Coronary artery bypass grafting; CAD: Coronary artery disease; CPB: Cardiopulmonary bypass;; CT: Computed tomography; CTA: Computed tomography angiography; MRI: Magnetic resonance imaging: TTE: Transthoracic echocardiography 


\section{Acknowledgements}

Not applicable.

\section{Availability of data and material}

Data sharing not applicable to this article as no datasets were generated or analyzed during the current study.

\section{Authors' contributions}

RX and KL designed the study, performed the operation, and revised the manuscript critically. JX, HP, YW and BL collected the data and wrote the article. DW, TW, ZZ and DL referred to the related literature. All authors read and approved the final manuscript.

\section{Funding}

No funding sources.

\section{Ethics approval and consent to participate}

Not applicable.

\section{Consent for publication}

Not applicable.

\section{Competing interests}

The authors declare that they have no competing interests.

Received: 1 July 2019 Accepted: 20 September 2019

Published online: 15 November 2019

\section{References}

1. Thomas JE, Eror AT, Kenney M, Caravalho J Jr. Asymptomatic right atrial cavernous hemangioma: a case report and review of the literature. Cardiovasc Pathol. 2004;13(6):341-4 https://doi.org/10.1016/..carpath.2004.09.001.

2. Burke A, Virmani R. Vascular tumors and tumor-like conditions. In: Atlas of tumor pathology. Tumors of the heart and great vessels. Burke A, Virmani R, editors. Washington, DC: Published by the Armed Forces Institute of Pathology; Be-thesda, MD; 1996. p. 79-90.

3. Patel J, Sheppard MN. Sudden death owing to right atrial hemangioma.J Forensic Sci. 2011;56(2):529-30 https:/doi.org/10.1111/j.1556-4029.2010.01667.x.

4. Novitzky, D., Guglin, M., \& Sheffield, C. (2009). Cardiac autotransplantation for removal of left atrial hemangioma and a review of the literature. In the heart surgery forum (Vol. 12, no. 5, pp. E279-84). https://doi.org/10.1532/ HSF98.20091027

5. Matsumoto Y, Watanabe G, Endo M, Sasaki H. Surgical treatment of a cavernous hemangioma of the left atrial roof. Eur J Cardiothorac Surg. 2001; 20:633-5 https://doi.org/10.1016/s1010-7940(01)00856-9.

6. Gersak B, Sostaric M, Dolenc-Strazar Z, Staric F, Kozelj M. Cavernous hemangioma in the junction between the left atrium and the aorta: case report. Heart Surgery Forum. 2005:8(1):72-4 https://doi.org/10.1532/HSF98.20041124.

7. Samanidis G, Khoury M, Balanika M, Dimitriou S, Michalis A. Cardiac cavernous hemangioma: diagnosis and treatment. ANZ J Surg. 2014;84(4): 294-5 https://doi.org/10.1111/ans.12256.

8. Ouerghi, S. , Youssef, A. B. , Ouechtati, W. , Zidi, A. , Mezni, F. , \& Belhani, A. , et al. (2011). Cardiac cavernous haemangioma. Heart Lung \& Circulation, 20(3), 0-201. https://doi.org/10.1016/.hlc.2010.08.013

\section{Publisher's Note}

Springer Nature remains neutral with regard to jurisdictional claims in published maps and institutional affiliations. 